D Nikčević, D Terzić, B Dupanović, B Andrić. Clinic for Infectious Diseases, Clinical Center of Montenegro, Podgorica, Montenegro

Background and Aims One third of the world population has been infected by the hepatitis B virus (HBV), causing an enormous worldwide burden of chronic hepatitis, cirrhosis and hepatocellular carcinoma. We make revue of clinical characteristics of acute hepatitis B infection in children in Montenegro.

Methods 180 children suffering from acute virus hepatitis were examined on Clinic for infectious diseases in Podgorica during the period 2006-2012. We used epidemiological and clinical data and blood tests for hepatitis viruses, Epstein-Barr virus, CMV and adenoviruses. Research sample is consisted of 18 children, which are old from 7-15 years with clinical, laboratory and virology verification of acute hepatitis $B$.

Results Epidemiological data have indicated the infection trough blood or blood products in 14 children and in 4 the way of infection remained unknown. HBsAg and antiHBc IgM were positive, values of alanine aminotransferase were elevated and PCR HBV DNK was higher than $200000 \mathrm{U} / 1$ in all children. The most frequent symptoms were the loss of appetite (88\%) and fatigue (70\%). Fever was registered in $30 \%$ of children, while the diarrhea is reported in $25 \%$, hepatomegaly in $86 \%$, enlarged spleen in $20 \%$ and icterus in $60 \%$ of cases. Patients without symptoms were present in $12 \%$ of cases. Three children after a follow-up longer than one year developed chronic infection.

Conclusions Even though immunisation for hepatitis B virus is mandatory since 2002 in Montenegro, the acute hepatitis B isn't rare infection. Children are at the greatest risk for developing potentially fatal complications of the infection.

\section{EPIDEMILOGIC STUDY ON PATHOGENIC BACTERIA CAUSING DIARRHEA IN CHILDREN AND DETECTION OF ANTIBIOTICS RESISTANCE PATTERNS IN HAMADAN, WEST OF IRAN}

doi:10.1136/archdischild-2012-302724.0939

'R Yousefimashouf, ${ }^{2} \mathrm{~F}$ Eghbalian, ${ }^{3} \mathrm{M}$ Koshki. 'Department of Microbiology; ${ }^{2}$ Dept. of Pediatrics; ${ }^{3}$ Hamadan University of Medical Sciences, Hamadan, Iran

Background and Aim Bacterial diarrhea is very common in particular in devoloping countries and is still one of the most causes of mortalities in children. The aim of present study was to identify the most common of bacterial agents causing acute bacterial diarrhea in children under 14 years old and detection of their resistance to antibiotics.

Methods During two years, 610 samples obtained from children under 14 years old with gastroentrotitis were investigated for bacterial cultures, frequency of age, serogrouping of isolates and antibiogram patterns. Polyvalent (I, II, III, IV) and monovalant antisera were used for serogrouping of E. coli (EPEC). Antibiogram tests were also performed by gel-diffusion method of Kirby-Bauer. The data were gathered through a questionnaire and analysed using spss software.

Results Out of 610 tested samples, 155 cases (25.4\%) had positve culture for intestinal pathogenic bacilli. The most common isolate was; Escherichia coli (EPEC) with 105 cases (67.8\%) and the lowest isolate was Shigella with 18 cases $(11.6 \%)$. The most common serogroup of E. coli was $0128(26.6 \%)$ and the lowest serogroup was 0119 $(5.7 \%)$. The most common serogroups of Salmonella were S. typhi $(34.4 \%)$ and $S$. typhimurium. The most common serogroup of Shigella was $S$. sonnei $(55.6 \%)$. The most effective antibiotics against bacteria were ceftriaoxne, nitrofurantoin, imipenem, amikacin and gentamycin.

Conclusions The present study showed that Escherichia coli (EPEC) and Salmonella species are predominant causes of bacterial diarrhea in children under 14 years old in this region. Most species showed high resisitance to routine antibiotics such as ampicillin, trimethoprim and chloramphenicol.

\section{CLINICAL SPECTRUM OF RESPIRATORY TRACT INFECTIONS: A STUDY AMONG CHILDREN IN AJMAN, UAE}

doi:10.1136/archdischild-2012-302724.0940

${ }^{1} \mathrm{M}$ Hassan, ${ }^{2} \mathrm{LJ}$ John, ${ }^{3} \mathrm{M}$ Hassan. 'Pharmaceutics; 2 Pharmacology, Gulf Medical University; ${ }^{3}$ Pediatrics, Gulf Medical College Hospital, Ajman, United Arab Emirates

Background and Aims Respiratory tract infection (RTI) among children is one of the most common reasons for physician visits and hospitalization and associated with significant morbidity. It is a leading cause of sickness among school children and parental absenteeism from work. Accordingly, this study was conducted to evaluate the spectrum of the respiratory tract infections among the children attending the outpatient department (OPD) of pediatrics in GMC Hospital, Ajman.

Methods A cross-sectional study was conducted from the medical records of pediatric patients (aged 0-12 years) diagnosed with RTIs during January 2011 at GMC Hospital, Ajman. The demographic data and the clinical diagnosis were analyzed (descriptive statistics) using SPSS version 19.

Results A total of 2416 patients attended the OPD of pediatrics during the study period of which RTI accounted for $20.2 \%$ ( $n=488)$. $55.5 \%(n=271)$ were males and $44.5(n=217)$ were females. Majority of the patients were Egyptians followed by Emiratis and Indians. Forty six percent of the patients (225) were between 1-5 years of age. The four most commonly diagnosed RTIs were unspecified upper respiratory tract infections (URTI) (20.3\%), common cold combined with bronchitis (17.8\%), tonsillitis (8.8\%), followed by bronchitis (8\%) in rank order. URTI was most commonly presented by children of $1-5$ years $(50.5 \%)$, while common cold combined with bronchitis was frequent among those below 1 year (47.1\%).

Conclusions Comparable to previous reports upper respiratory tract infections were the most common RTI among this population and children of 1-5 years were most vulnerable to RTI

\section{AUTONOMIC DYSFUNCTION IN INFANTS WITH RSV INFECTION}

doi:10.1136/archdischild-2012-302724.0941

${ }^{1} \mathrm{C}$ Liebrand, 'M Bouwman, ${ }^{2} \mathrm{I}$ Ahout, ${ }^{3} \mathrm{M}$ Kox, ${ }^{1} \mathrm{C}$ Neeleman. ${ }^{1}$ Pediatric ICU; ${ }^{2}$ Department of infectious diseases, ${ }^{3} I C U$ research department, UMCN St Radboud, Nijmegen, The Netherlands

Background Apnea can be a presenting symptom in respiratory Synctial Virus (RSV) infection. The incidence of apnea in RSV infected infants varies between 16 and $25 \%$ with a particularly high risk associated with young age $(<3 \mathrm{mo})$ and prematurity. Apnea is frequently observed in early RSV infection when symptoms are still confined to the upper airways, suggesting inflammatory response induced autonomic dysfunction.

Objective The aim of the study is to assess autonomic dysfunction as indexed by Heart Rate Variability (HRV) in severe RSV infection. The secondary parameter is to analyze the effect of other viral pathogens on autonomic function in infants with LRTI requiring mechanical ventilation.

Methods Prospective observational study in a tertiary PICU in infants with viral LRTI requiring mechanical ventilation. Controls were age matched infants without signs of infection. Autonomic function was assessed via HRV, monitoring low frequency (LF) and high frequency (HF) indices of short time recordings (5 min).

Results In 7 patients and 13 controls 36 recording were analyzed. Total HRV, LF and LF/HF ratios were significant lower in RSV patients versus controls $(p<0.0045, p<0.0002$ and $p<0.0001$ respectively). In ventilated infants with LRTI caused by other respiratory 